\title{
DNA-templated dimerization
}

This is the first time a DNA catalyst has been used in natural product total synthesis
Dictazole B is one member of the aplysinopsin family of marine natural products. The unusual spiro-fused cyclobutane core of the structure is thought to be formed in nature by a photocatalysed [2+2] cycloaddition between two derivatives of the parent natural product (E)-aplysinopsin. Now, a team of French and UK researchers have shown that DNA could template this cycloaddition process in aqueous buffer, leading to an improved biomimetic synthesis of dictazole $B$.

"We have, for some time now, had an ongoing collaboration with the group of Michael Smietana - to extend the scope of DNA-mediated catalysis," explains Arseniyadis. "We became interested in whether DNA could be used to bring reagents together and promote otherwise challenging reactions. A synthesis of dictazole B reported by Laurent Evanno, Erwan Poupon and co-workers offered us the perfect opportunity to explore this concept in the context of natural product synthesis."

Poupon, Evano and co-workers had first reported a biomimetic total synthesis of dictazole B in 2014, but the key photodimerization step only worked in the solid state and the reaction could not easily be scaled up. "We wanted to see if DNA could effectively template a putative [2+2] cycloaddition, whether it would work selectively for the heterodimerization that leads to dictazole $B$ and whether the chirality of the DNA would lead to an enantioenriched product," says Arseniyadis.

As a first step, the team investigated a homodimerization reaction. Irradiation of a solution of (E)-aplysinopsin in aqueous buffer in the presence of salmon testes DNA (commercially available random DNA sequences) over 4 days afforded

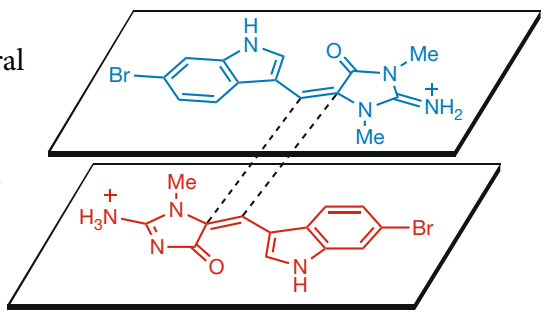

a $23 \%$ yield of the head-to-tail dimerized product. In contrast, when DNA was omitted, only degradation of the starting material was observed, presumably through a $[2+2]$ cycloaddition of singlet oxygen onto the double bond followed by a rapid fragmentation.

To rationalize the results of this DNA-templated process, a series of circular dichroism (CD) experiments were performed. Substoichiometric additions of aplysinopsin to DNA result in no significant changes in the CD spectrum. The addition of between 1 and 2 equivalents of the substrate, however, leads to changes that indicate a slight unwinding of the DNA, and the appearance of signals that are indicative of groove binding and a disymmetric interaction of two chromophores (the substrate).

Control experiments using pyrophosphate in place of DNA and using a neutral substrate (in place of the cationic aplysinopsin) gave no cycloaddition products and confirmed that both groove binding and electrostatic interactions are important for the observed reactivity. The necessary head-to-tail stacking of two cationic monomers is well-known for other cationic groove-binding molecules and so the team invoke such an interaction in their reaction by analogy.

The resulting model of the dimerization is that a combination of groove binding and electrostatics mediate the interaction of the first monomer with DNA. The formation of a head-to-tail aggregate

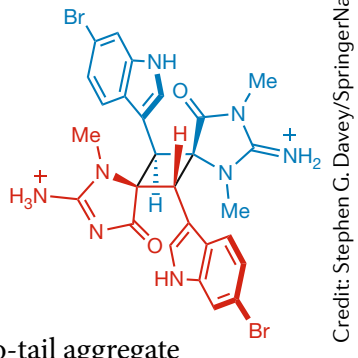

is then favoured to minimize repulsion between the monomers before the photocycloaddition affords the cyclobutane containing product.

The researchers went on to apply their optimized reaction conditions to the heterodimerization reaction necessary for the formation of dictazole B. "We were surprised and ecstatic to obtain a $16 \%$ yield of the natural product," says Arseniyadis. "Our DNA-mediated approach far exceeds the efficacy of the solid state reaction. This is the first time a DNA catalyst has been used in natural product total synthesis." Although the reaction only proceeds with low enantioselectivity (7\%), it is hoped this might be optimized by fine tuning both the reaction conditions and the DNA sequence.

"In the future, we'd like to extend our strategy and develop a more general method for the synthesis of cyclobutanes, and apply it to other cyclobutane-containing natural products. We'd also like to extend applications of DNA catalysis into photoredox chemistry," says Arseniyadis.

Stephen G. Davey

ORIGINAL ARTICLE Duchemin, N. et al. DNA-templated [2+2] photocycloaddition: a straightforward entry into the aplysinopsin family of natural products. Angew. Chem. Int. Ed. https://doi.org/10.1002/anie.201806357 FURTHER READING Skiredj, A. et al. Spontaneous biomimetic formation of $( \pm)$-dictazole $B$ under irradiation with artificial sunlight. Angew. Chem. Int. Ed. 53, 6419-6424(2014) 